\title{
Mecanismos de enfrentamento utilizados por idosos residentes em instituições de longa permanência
}

\author{
Coping mechanisms used by elderly residents \\ in long-stay institutions
}

\section{Mecanismos de afrontamiento utilizados por los residentes de edad avanzada en instituciones de larga estadía}

Recebido: 26/07/2013

Aprovado: 13/01/2014

\author{
Jurema Ribeiro Luiz Gonçalves ${ }^{1}$ \\ Gabriela Carvalho da Silva ${ }^{2}$ \\ Erica Aparecida dos Santos ${ }^{3}$ \\ Pollyana Pagliaro Borges Soares ${ }^{3}$ \\ Carla Maria Silvano ${ }^{4}$ \\ Eliseu da Costa Campos ${ }^{5}$
}

Esta pesquisa teve como objetivo identificar as estratégias de enfrentamento utilizadas como mecanismo de adaptação por idosos institucionalizados. Trata-se de um estudo qualitativo, descritivo e exploratório, desenvolvido em uma instituição de longa permanência para idosos (ILPI) no interior de Minas Gerais. Da população de 48 idosos, cinco não tiveram declínio cognitivo, segundo o Mini Exame do Estado Mental (MEEM). Destes, três aceitaram participar da pesquisa. As falas gravadas foram transcritas. Os resultados mostraram predomínio do sexo masculino, com faixa etária de 60 a 69 anos, sendo a maioria dos entrevistados solteira e com renda de um salário mínimo. Foram observadas as seguintes categorias referentes às estratégias de enfrentamento: Participação nas atividades diárias da ILPI e Aceitação frente à ausência de atividades de lazer. A adaptação deu-se por meio da participação em atividades de rotina da ILPI, fazendo com que se sentissem úteis e aceitos no âmbito asilar.

Descritores: Adaptação, Idoso, Instituição de Longa Permanência para Idosos.

This research aimed to identify the coping strategies used as adaptation mechanism for institutionalized elderly. This is a qualitative, descriptive and exploratory study, developed in a home for the aged in Minas Gerais, Brazil. The population is 48 elderlies, and five had no cognitive decline according to the Mini Mental State Examination. Of these, accepted to participate in the research only three elderlies. The recordings were transcribed. The results showed a predominance of males, aged 60 to years, singles and income of a minimum wage. It was observed the following categories related to coping strategies: Participation in daily activities of a home for the aged and Acceptance forward to the absence of leisure activities. The adaptation was favored for by participation in routine activities of home for aged, making them feel useful and accepted under asylum.

Descriptors: Adaptation, Aged, Homes for the Aged.

Esta investigación tuvo como objetivo identificar las estrategias de afrontamiento utilizadas como mecanismo de adaptación para personas mayores institucionalizadas. Se trata de un estudio cualitativo, descriptivo y exploratorio, desarrollado en una institución para ancianos en Minas Gerais, Brasil. Tenido como población 48 adultos mayores, de los cuales cinco no tenían deterioro cognitivo según el Mini Examen del Estado Mental. Tres de ellos aceptaron participar. Las líneas grabadas fueron transcritas. Los resultados mostraron un predominio del sexo masculino, con edades entre 60 a 69 años, solteros y que ganaban un salario mínimo. Se Observó las siguientes categorías relacionadas con las estrategias de afrontamiento: Participación en las actividades diarias de la institución y Aceptación adelante a la falta de actividades de ocio. La adaptación se llevó a cabo mediante la participación en actividades rutinarias, haciendo que se sientan útiles y aceptados en la institución.

Descriptores: Adaptación, Anciano, Hogares para Ancianos.

1. Enfermeira. Doutora em Enfermagem. Professora Adjunta II - Departamento de Enfermagem em Educação e Saúde Comunitária e Pós Graduação em Atenção à Saúde da Universidade Federal do Triângulo Mineiro - UFTM. Coordenadora do Projeto. juremaluiz@ig.com.br

2. Enfermeira pela UFTM.

3. Enfermeira. Mestre em Atenção à Saúde pela UFTM.

4. Enfermeira. Especialista em Saúde do Idoso - Modalidade Residência Multiprofissional. Mestranda em Ciências da Saúde pelo Instituto de Assistência Médica ao Servidor Público Estadual de São Paulo - IAMSPE.

5. Enfermeiro. Mestrando em Atenção à Saúde pela UFTM. 


\section{INTRODUÇÃO}

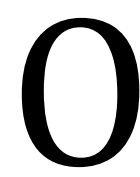

aumento da população idosa é uma realidade no Brasil. Em 1960, o Brasil era composto por três milhões de idosos, já em 1975, esse número aumentou para sete milhões, e em 2008, aumentou ainda mais, chegando a 20 milhões de idosos. Em decorrência desse aumento, as doenças próprias do envelhecimento começaram a ganhar maior interesse de expressão na sociedade ${ }^{1}$.

Nos dias atuais, a longevidade segue junto à qualidade de vida, havendo um olhar a mais para a saúde e o bem-estar da pessoa idosa, por sua vez a longevidade também se relaciona a presença de agravos á saúde. A qualidade de vida não está interligada somente ao aparecimento de doenças, mas também, em proporcionar bem-estar físico, psicológico, funcional e social para as pessoas idosas ${ }^{2}$.

Para proporcionar qualidade de vida aos idosos institucionalizados, além do carinho e da atenção da instituição, é necessária a presença de pessoas próximas, como amigos e familiares, a fim de evitar a solidão e o isolamento social dessa população².

A entrada de idosos em instituições de longa permanência para idosos (ILPI) é vista como opção para os que não conseguem viver sozinhos ou não têm familiares, ou ainda, para aqueles que a família não tem como sustentar dentro de suas casas. Historicamente, há um estigma com relação à institucionalização, a qual era vista como abrigo para idosos pobres e sozinhos ou doentes mentais. Hoje, a institucionalização pode ser vista por alguns idosos e familiares como a busca de um envelhecimento digno ${ }^{3}$.

Uma das principais questões quando se trata de institucionalização é a busca da adaptação do idoso, pois esse processo pode diminuir com o aumento da idade. Tal fato influencia para que haja um aumento no desajuste com a instituição ao longo dos anos $^{2}$.
Quando é realizada a admissão do idoso em uma ILPI, ocorre um impacto na sua vida. Consequentemente, há mudanças de hábitos na sua rotina, no seu espaço, nas relações interpessoais e em seus costumes, constituindo um fator de predisposição à alteração do comportamento, fazendo com que o idoso faça um esforço significativo para desenvolver estratégias de enfrentamento da realidade . $^{\text {. }}$

Esse processo de adaptação está relacionado à sua vida antes de ingressar na ILPI e é influenciado pelos seguintes fatores: onde o idoso residia, sua forma de apoio, sua liberdade de se expressar e seu espaço pessoal. Assim, seu processo adaptativo está interligado com o que ele vivenciou antes, bem como seu modo de lidar com as mudanças nesse processo de saída de um ambiente familiar para um ambiente estranho.

Em razão das particularidades que o idoso leva consigo para uma ILPI, existe uma expressiva variabilidade na experiência de eventos de sua vida, marcados por serem estressores, e nas formas de enfrentamento destes. Isso os leva a apresentar diferentes formas de enfrentar esses eventos.

Os mecanismos de enfrentamento podem ter efeito de amortecer as condições adversas, ou seja, aquelas que eles têm que enfrentar no decorrer da sua existência, como perdas e danos aos sentimentos. Isso pode ser benéfico para o idoso porque o faz sentir que há um meio de enfrentar os fatores estressantes ${ }^{6}$.

0 idoso que utiliza o mecanismo de enfrentamento pode vivenciar um aumento do sentimento de controle sobre si próprio e sobre o que o cerca, um aumento da autoestima, uma maior capacidade de se colocar no lugar do outro. Ou seja, empatia e aumento da proximidade com outras pessoas, criando-se assim um vínculo amigável, mudanças nos valores e maior entendimento e conhecimento de si e dos outros ao seu redor 6 . 
Além de os idosos vivenciarem dificuldades adaptativas ao serem institucionalizados, observa-se que os profissionais de enfermagem inseridos nesse âmbito não estão preparados para cuidar dos aspectos emocionais dessa população, focando apenas nos cuidados físicos ${ }^{7}$. Em razão disso, é necessário entender as queixas e dificuldades emocionais dos idosos para que se possa planejar um cuidado humanizado com foco, também, na saúde mental destes. Este estudo tem como objetivo identificar as estratégias de enfrentamento utilizadas como mecanismo de adaptação por idosos de uma ILPI.

\section{MÉTODO}

Trata-se de um estudo qualitativo, descritivo e exploratório, desenvolvido em uma ILPI de caráter filantrópico localizada na cidade de Uberaba-MG. A instituição foi escolhida por ser vinculada à Universidade Federal do Triângulo Mineiro (UFTM) para a realização de estágios e projetos.

Inicialmente, contatou-se a coordenadora da ILPI para esclarecer os objetivos do estudo e solicitar o consentimento para iniciar a pesquisa. Após a aprovação, foi obtida uma listagem das pessoas idosas residentes na instituição para que fosse possível a aproximação com os eventuais participantes da pesquisa.

Os critérios de inclusão para participar da pesquisa foram: ter interesse em participar do estudo, ter 60 anos ou mais, não apresentar declínio cognitivo segundo o Mini Exame do Estado Mental (MEEM) e ter aceitado assinar ou carimbar as digitais (em caso de analfabetismo) no Termo de Consentimento Livre e Esclarecido (TCLE). Dos 48 idosos residentes na ILPI, apenas cinco não tinham declínio cognitivo e, destes, três aceitaram participar da pesquisa.

Foram utilizados os seguintes instrumentos: 1) avaliação socioeconômica e demográfica. Utilizou-se parte do questionário Older Americans Resources and Services (OARS), adaptado por Ramos ${ }^{8}$ na versão brasileira. Com base nesse instrumento, selecionaram-se os seguintes itens: sexo; faixa etária (60-70, 70-80, 80 ou mais); situação conjugal \{(nunca se casou ou morou com companheiro(a)/mora com esposo(a) ou companheiro(a)/viúvo(a), separado(a), desquitado(a) ou divorciado(a)\}; escolaridade (em anos); e renda individual mensal (em salários mínimos); 2) MEEM para a avaliação cognitiva dos idosos que tenham 60 anos ou mais, que permite a avaliação da função cognitiva e o rastreamento de quadros demenciais. Esse instrumento foi desenvolvido por Folstein ${ }^{9}$ e adaptado à realidade brasileira por Bertolucci ${ }^{10}$, sendo composto por questões que avaliam orientação, memória imediata e de evocação, concentração, cálculo, linguagem e domínio espacial, com escore total de 30 pontos. 0 ponto de corte será considerado de acordo com a escolaridade, sendo que idosos analfabetos devem ter pontuação $\leq 13$; os que tem de 1 a 11 anos de estudo, $\leq 18$; e os que tem escolaridade maior que 11 anos, $\leq$ maior ou igual a 26 anos $^{9-11}$.

A coleta de dados foi realizada nos meses de julho a agosto de 2012. Os entrevistados foram identificados por um número para preservar a confidencialidade dos dados. Foi realizada uma entrevista utilizando-se a seguinte questão norteadora: "O que o(a) senhor(a) fez para se adaptar a essa nova experiência de estar residindo em uma ILPI?".

As falas foram gravadas $\mathrm{e}$ transcritas para o programa de texto Word $\AA$ para Windows 7. Para o tratamento dos dados obtidos, utilizou-se a Análise de Conteúdo ${ }^{12}$, seguindo os seguintes passos: ordenação dos dados referente às entrevistas e transcrição das fitas cassetes; releitura do material e organização dos relatos; classificação dos dados, com releitura exaustiva e repetida dos textos; constituição de um "corpus" de comunicações; leitura transversal de cada corpo com recorte de "unidades de registro"; classificação por temas mais 
relevantes; análise final, considerando os objetivos do trabalho, o referencial teórico, os temas que emergiram dos depoimentos dos profissionais; e a utilização dos registros contidos no diário de campo ${ }^{12}$.

0 projeto foi encaminhado e aprovado pelo Comitê de Ética em Pesquisa da Universidade Federal do Triângulo Mineiro - UFTM sob o protocolo 2236/12.

\section{RESULTADOS}

Os idosos entrevistados apresentaram as seguintes condições socioeconômicas e demográficas: dois do sexo masculino e uma do sexo feminino; dois com idade entre 60 e 69 anos, e um, entre 70 e 79 anos; dois solteiros e um divorciado; um sem escolaridade, um com quatro a oito anos de estudo, e um com oito anos de estudo, sendo que todos os entrevistados tinham um salário mínimo como renda individual.

Com base nas entrevistas realizadas, foram observadas as seguintes categorias referentes às estratégias de enfretamento como mecanismo de adaptação: Participação nas atividades diárias da ILPI e Aceitação frente à ausência de atividades de lazer.

\section{Participação nas atividades diárias da ILPI}

A adaptação foi facilitada pela ajuda prestada na rotina da instituição como forma de passar o tempo, pela interação social e pelo conhecimento do novo ambiente, sendo utilizada positivamente pelos idosos. Esses fatores são observados nas falas:

[...] Ajudo a recolher os pratos, ajudo a levar uma cega no banheiro, ajudo um pouquinho ali, aqui. (E1)

[...] Faço limpeza, ajudo a lavar quando precisa. (E3)

\section{Aceitação frente à ausência de atividades de lazer}

Verifica-se a ausência de atividades de lazer dentro da ILPI, levando os idosos ao conformismo em relação à realidade local. [...] Aqui não tem nada pra gente fazer, não tem um jogo, não tem diversão, não tem nada, o único jogo que a gente divertir aqui é escutando um rádio, vendo televisão, dia de jogo, a gente gosta de futebol, aí a gente vê, mas no resto não tem nada não [...]. (E2)

[...] Não tô querendo ficar aqui mais não. Só faço isso (auxilia na limpeza). (E3)

Dentre os entrevistados, um se destacou ao se referir aos passeios semanais com os familiares como forma de lazer:

[...] Às vezes eu ando, eu saio. Eu tenho umas primas minhas que me busca no fim de semana e me leva pra casa delas [...]. (E2)

\section{DISCUSSÃO}

Frente aos resultados encontrados, percebe-se que há relatos de um estudo ${ }^{13}$ que reafirmam os achados desta investigação, uma vez que, para alguns idosos, o fato de ajudar nas atividades diárias faz com que eles se sintam úteis e produtivos, tornando a institucionalização mais aceitável.

Investigação realizada em Fortaleza ${ }^{14}$ mostrou que o trabalho doméstico inicial dentro da instituição favorece a adaptação do idoso. Muitos se sentem felizes e bem adaptados em virtude dessa interação idoso/comunidade na instituição.

Em relação à segunda categoria, pesquisa realizada com idosas institucionalizadas da região norte do Rio Grande do Sul mostrou que a estratégia utilizada para enfrentar o asilamento e o processo natural do envelhecimento foi ocupar a maior parte do tempo assistindo televisão, assim como relatado no estudo em questão. Outras idosas, que residiam em instituições de livre acesso, preferiam passear. Essa possibilidade dada aos que possuíam condições físicas adequadas favoreceu a quebra da rotina na instituição, permitindo controle do tempo, satisfação e independência do idoso ${ }^{13}$.

Observou-se durante as entrevistas que os idosos apresentavam-se tristes e conformados por não terem o que fazer no local, principalmente em relação a atividades de lazer. 0 conformismo pode estar relacionado à necessidade de sobrevivência que, apesar de não garantir o 
lazer e conforto, garante os cuidados essenciais que muitos idosos institucionalizados precisam para ter um envelhecimento mais digno ${ }^{15}$. Os poucos momentos de satisfação expressados pelos idosos estavam ligados a visitas e passeios realizados com os familiares nos finais de semana.

O enfermeiro dentro da ILPI atua no cuidado de forma a não causar danos ao longo do processo de adaptação do idoso, ou seja, evitando a diminuição da autonomia, reforçando a identidade e a socialização, assim como a qualidade de vida. As ILPIs devem atuar no processo saudável do envelhecimento, promovendo atividades que tragam ânimo e satisfação aos idosos. Isso pode acontecer envolvendo o próprio idoso no planejamento das atividades ${ }^{4}$.

Uma forma de auxiliar o idoso nesse processo de adaptação é utilizando atividades lúdicas nos cuidados prestados a ele. Esse tipo de ação traz a oportunidade de despertar a criatividade, a imaginação, os sentimentos alegres e o relacionamento interpessoal à sua volta, além de ajudar na melhora da saúde e no relacionamento do grupo dentro da instituição ${ }^{16}$.

\section{CONCLUSÃO}

Para os idosos entrevistados, a adaptação deu-se por meio da participação em atividades de rotina da ILPI, fazendo com que se sentissem úteis, favorecendo a aceitação asilar. Observou-se, também, que a adaptação foi desfavorecida pela pouca oferta/participação em atividades de lazer. Apesar disso, algumas formas de entretenimento, como televisão, rádio e passeio, mostraram-se determinantes para mudar a rotina dos idosos e promover interação com os outros residentes.

Nessa realidade, a enfermagem pode trabalhar os aspectos físicos, emocionais e sociais dos idosos institucionalizados segundo a demanda referida por eles próprios. Neste caso, foi relatada a necessidade de se realizarem atividades diferentes, que façam com que os idosos fujam à rotina do local. 0 enfermeiro como agente promotor de saúde, pode trabalhar com atividades lúdicas e recreativas com objetivo terapêutico, visando à melhor adaptação do idoso em seu novo lar.

\section{REFERÊNCIAS}

1. Veras R. Envelhecimento populacional contemporâneo: demandas, desafios e inovações. Rev Saúde Pública [Internet]. 2009; 43(3):548-54 [citado em 15 fev 2013]. Disponível em: http://www.scielo.br/pdf/rsp/2009nahead/ 224.pdf

2. Carvalho P, Dias O. Adaptação dos idosos institucionalizados. Millenium [Internet]. 2011; 40:161-84 [citado em 26 mar. 2013]. Disponível em: http://www.ipv.pt/millenium/Millenium40/ 12.pdf.

3. Lenardt $\mathrm{MH}$, Michel T, Wachholz PA. Autoavaliação da saúde e satisfação com a vida de idosas institucionalizadas. Cienc Cuid Saúde [Internet]. 2010; 9(2):246-54 [citado em 24 abr 2013]. Disponível em: http://periodicos.uem.br/ojs/index.php/Cie ncCuidSaude/article/view/8041/6073.

4. Clos MB. Recusa, conformidade e libertação: considerações sobre o processo de adaptação de idosos em uma instituição de longa permanência para idosos. [dissertação na internet] Porto Alegre (RS): Universidade Federal do Rio Grande do Sul. Faculdade de Educação; 2010 [citado em 22 maio 2013]. 113p. Disponível em: http://www.lume.ufrgs.br/handle/10183/2 8800

5. Fortes-Burgos ACG, Néri AL, Cupertino APFB. Eventos estressantes, estratégias de enfrentamento, auto-eficácia e sintomas depressivos entre idosos residentes na comunidade. Psicol Reflex Crit. [Internet]. 2008; 21(1):77-82 [citado em 26 mar 2013]. Disponível em: http://www.scielo.br/scielo.php?pid=s010279722008000100010\&script=sci_arttext.

6. Aldwin C, Sutton K, Lachman M. The development of coping resources in adulthood. J Personal. [Internet]. 1996; 64:837-71 [citado em 08 abr 2013]. Disponível em: 
http://www.ncbi.nlm.nih.gov/pubmed/8956 5

7. Silva BT, Santos SSC, Silva MRS, Sousa LD. Percepção das pessoas idosas sobre a institucionalização: reflexão acerca do cuidado de enfermagem. Rev Rene [Internet]. 2009; 10(4):118-25 [citado em 09 ago 2013]. Disponível em: http://www.revistarene.ufc.br/revista/index .php/revista/article/view/595

8. Ramos LR. Growing old in São Paulo, Brazil: assessment of health status and family support of the elderly of different socioeconomic strata living in the community. [tese]. London (UK): London School of Hygiene and Tropical Medicine, University of London; 1987. 327p.

9. Folstein MF, Folstein SE, Mchugh PR. "Mini-mental state". A practical method for grading the cognitive state of patients for the clinician. J Psychiatr Res. 1975; 12(3):18998.

10. Bertolucci PHF, Brucki SMD, Campacci SR, Juliano Y. O Mini Exame do Estado Mental em uma população geral: impacto da escolaridade. Arq Neuro-psiquiatr.[Internet]. 1994; 52:1-7. Disponível em: http://www.scielo.br/pdf/anp/v52n1/01.pd f

11. Saliba 0, Garbin CAS, Garbin AJI, Dossi AP. Responsabilidade do profissional de saúde sobre a notificação de casos de violência doméstica. Rev Saúde Pública [Internet]. 2007; 41(3):472-7 [citado em 23 abr 2013]. Disponível em: http://www.scielo.br/scielo.php?script=sci_a rttext\&pid=S0034-89102007000300021

12. Bardin L. Análise de conteúdo. 3aㅡ Ed, $2^{\underline{a}}$ reimpressão. Lisboa: Edições 70; 2007. 223p. 13. Pavan FJ, Meneghel SN, Junges JR. Mulheres idosas enfrentando a institucionalização. Cad Saúde Pública [Internet]. 2008; 24(9):2187-90 [citado em 3 maio 2013]. Disponível em: http://www.scielo.br/pdf/csp/v24n9/25.pdf 14. Bessa MEP, Silva MJ. Motivações para o ingresso dos idosos em instituições de longa permanência e processos adaptativos: um estudo de caso. Texto e Contexto Enferm. [Internet]. 2008; 17(2):258-65 [citado em 8 abr 2013]. Disponível em: http://www.scielo.br/scielo.php?script=sci_a rttext\&pid=S0104-07072008000200006
15. Marin MJS, Miranda FA, Fabbri D, Tinelli LP, Storniolo LV. Compreendendo a história de vida de idosos institucionalizados. Rev Bras Geriatr Gerontol. [Internet]. 2012; 15(1):147-54 [citado em 29 ago 2013]. Disponível em: http://www.scielo.br/scielo.php?script=sci_a rttext\&pid=S180998232012000100016\&lng $=$ n. $\quad$ http://dx.doi.org/10.1590/S180998232012000100016

16. Leite BFT, Salvador DHY, Araújo CLO. Avaliação cognitiva dos idosos institucionalizados. Rev Kairós [Internet]. 2009; 12(1):247-56 [citado em 24 abr 2013]. Disponível em: http://revistas.pucsp.br/index.php/kairos/a rticle/viewFile/2790/1825

\section{CONTRIBUIÇÕES}

Jurema Ribeiro Luiz Gonçalves coordenou o projeto, fez revisão crítica e participou da redação;

Gabriela Carvalho da Silva atuou na coleta de dados e redação;

Erica Aparecida dos Santos, Pollyana Pagliaro Borges Soares, Carla Maria Silvano e Eliseu da Costa Campos participaram na coleta e análise dos dados e redação final. 